\title{
VLSI INTERCONNECTION MODELLING USING A FINITE ELEMENT APPROACH
}

\author{
UMESH KUMAR \\ Senior Member, IEEE; Fellow, IETE.
}

\begin{abstract}
In the last decade, an important shift has taken place in the design of hardware with the advent of smaller and denser integrated circuit packages. Analysis techniques are required to ensure the proper electrical functioning of this hardware. An efficient method is presented to model the parasitic capacitance of VLSI (very large scale integration) interconnections. It is valid for conductors in a stratified medium, which is considered to be a good approximation for the $\mathrm{S}_{i}-\mathrm{S}_{i} \mathrm{O}_{2}$ system of which present day ICs are made. The model approximates the charge density on the conductors as a continuous function on a web of edges. Each base function in the approximation has the form of a "spider" of edges. Here the method used [1] has very low complexity, as compared to other models used previously [2], and achieves a high degree of precision within the range of validity of the stratified medium.
\end{abstract}

\section{VLSI INTERCONNECTIONS: INTRODUCTION}

Today's high level of integration can lead to very large and complex systems with extremely small physical dimensions and highly coupled elements. The advantages for using the VLSI interconnections are following:

(a) Low cost due to large quantities processed.

(b) Small size.

(c) High reliability because all components are fabricated simultaneously and there is no soldered joint.

(d) Improved performance.

The type of analysis required for a particular system depends on its performance and purpose. A fundamental quantity that characterizes a particular interconnection technology is what we call the general impedance level. It is simply the loseless characteristic impedance of the "average" connection in the system

$Z_{g}=\sqrt{\frac{L}{C}}$

Typical values of $Z_{g}$ range from 5 to $200 \mathrm{ohms}$.

The analysis of digital system interconnections encompasses a wide spectrum of frequencies. The purpose of computer-aided electrical analysis is to ensure that the hardware at hand meets the electrical design criteria.

ANALYSIS FOR RESISTANCE COMPUTATIONS:

The purpose of the resistance computation for the interconnections and the ground and voltage supply planes is to determine whether the voltage drops along 
the conductors are within tolerable limits. As the spatial dimensions are reduced for VLSI, an increase in the resistance results and usually the current per unit area increases.

The geometries in the resistance problem can be very complicated, consisting of planes and wires having complex shapes. The macro-model, which results as a solution of the resistance problem for the $n$ terminal currents $I$ and potentials $V$, is simply

$I=G V$

Where $I, V$ are matrices of order $(n \times 1)$ and $G$ is a matrix of order $(n \times n)$. This is the indefinite short circuit conductance matrix.

A macro-model is basically a simplified terminal model that allows the analysis of even large systems. The $i$ th element of the current vector $I$ is found by integration of divergence $J=0$ or

$I_{i}=\int_{s} \bar{J} \cdot \hat{n} d s$

Where $J$ is the current diversity and $S$ is a closed surface including a source of current $I_{i}$.

We use $V$ to indicate the indefinite matrix potentials.

The best computational technique for finding $G$ depends on many factors, such as:

a) Required accuracy

b) Conductor shape

c) Size of the given problem

A quasi-two dimensional solution with a uniform current density along the thickness suffices for most resistance computations. The grid of Fig. 1(a) is used for finite differences and for some of the finite element solutions, while the

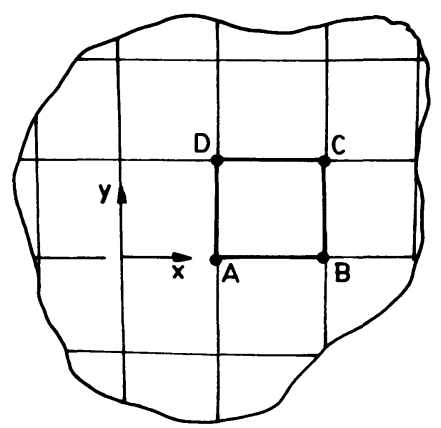

FIGURE 1 (a) Rectangular nodal grids 


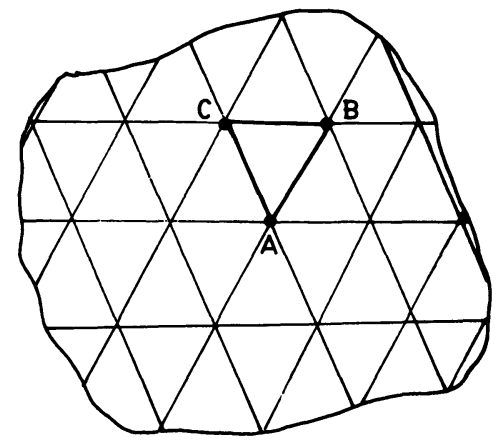

FIGURE 1 (b) Triangular nodal grids

triangular cells (Fig. 1(b)) are particularly suited for irregular boundary interfaces. The main difference for the various grids is the order of potential approximation, which is of the form

$V(x, y)=K_{1}+K_{2} x+K_{3} y+K_{4} x y$

An additional term is included in the above equation for each added node. As an illustration, we can match the potential at two points $A$ and $B$ by the cell $C_{i}$ shown in Fig. 2. The variation inside $C_{i}$ is $=$

$V(x, y)=((x B-x) /(x B-x A)) X V A$

$$
+((x-x A) /(x B-x A)) X V B
$$

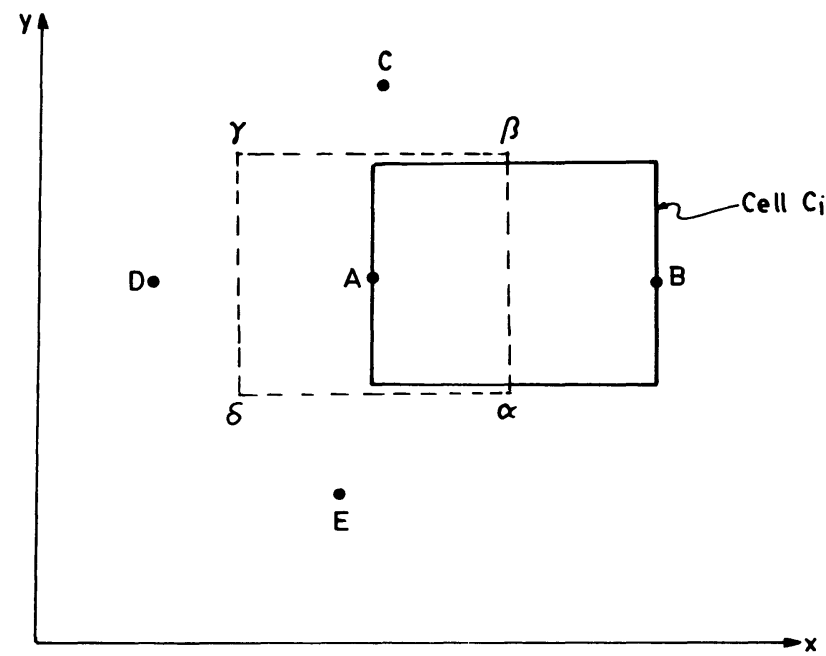

FIGURE 2 Rectangular cell and current surface 
The current through cell $C i$ is measured by the surface $a-b$ with a length ( $a b$ ) and the thickness $t$, with

$J=\sigma E=-\sigma\left(\frac{\partial V}{\partial x}\right)$

Thus, equation (2) becomes

$I_{i}=\left(\frac{1}{R}\right) \int_{a}^{b} \frac{\partial V}{\partial x} d y=-|a b| /(R) \frac{X \partial V}{\partial x}$

where the resistance per square is $R=1 / \sigma t, \sigma=$ conductivity, $t=$ thickness of the conductor. Finally, obtaining $\partial V / \partial x$ from equation (4)

$I_{i}=|a b| /(R(x B-x A))$

Where the conductance of the cell $C i$ is

$G_{A B}=|a b| /(R(x B-x A))$

The total surface for the current computation of equation (2) for node $A$ is bounded by $a-b-c-d$, as shown in the Figure 2, and the external current may be injected at node $A$. The modified nodal stamp that enters the appropriate contributions in the circuit matrix is

$\begin{array}{cccc} & V_{A} & V_{B} & R H S \\ A & G_{A B} & -G_{A B} & 0 \\ B & -G_{A B} & G_{A B} & 0\end{array}$

For node $A$ and all the neighboring nodes, the matrix stamp is

$$
\begin{array}{ccccccc} 
& V_{A} & V_{B} & V_{C} & V_{D} & V_{E} & R H S \\
& G_{A B}+G_{A C} & -G_{A B} & -G_{A C} & -G_{A D} & -G_{A E} & I_{i} \\
& G_{A D}+G_{A E} & & & & &
\end{array}
$$

The circuit matrixes formed by stamping in contributions of the type of equation (9) are extremely sparse and the solution of systems with several thousand unknowns is feasible.

\section{ANALYSIS FOR INDUCTANCE COMPUTATIONS:}

Integrated circuit geometries are more closely related to physically large power bus structures rather than the lumped coils used in electronic circuits. The computation for two-dimensional geometries such as long power buses or stripline-type geometries is possible. Three-dimensional integrated circuit inductances are computed via a building block approach. The building blocks, which are rectangular conductors, are called partial conductors. In this approach, the so-called partial 


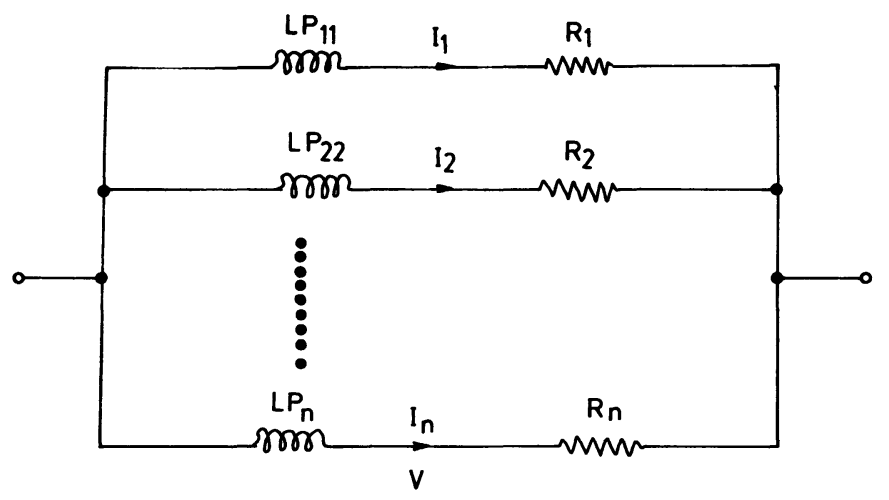

FIGURE 3 Equivalent circuit for inductance per unit length

self and partial mutual inductances are computed from:

$L_{p r m}=\frac{\mu_{0}}{4 \pi} \frac{1}{a_{k} a_{m}} \int_{a_{k}} \int_{a_{m}} \int_{b_{k}}^{c_{k}} \int_{b_{m}}^{c_{m}} \frac{\left|\overline{d l_{k}} \cdot \overline{d l m}\right|}{|\overline{r b k}-\overline{r m}|} d a_{k} d a_{m}$

Where the conductors $k$ and $m$ coincide for the partial self-inductances. The conductors are $a k$ and $a m$, while the length co-ordinates $b_{k}, b_{m}$ and $c_{k}, c_{m}$ represent the end points as shown in Fig. 3. The inductances in equation (10) are called partial inductances to distinguish them from the inductances of the loops and other arrangements.

If the conductor of Fig. 3 is reduced by ' $a$ ' in all dimensions, then its new partial inductance is $L_{p k m}=a L_{p k m}$.

For a system of partial conductor, we came up with the partial inductance matrix $L_{p}$, where in the $s$-domain

$V(s)=s L_{p} I(s)$

Inductances of the overall physical arrangement are computed using network analysis where $V, I$ are the branch voltages and current, respectively. If we specify a set of nodes in the partial inductance network, we compute the inductance from

$V_{i}(s)=s L_{p i j} I_{j}(s)$

Where $I_{k}=0$ for $k \neq j, L_{p}$ is a matrix of order $(n \times n)$.

Thus, all currents are set to zero, except for the source applied at the partial conductor $j$, while the voltage is measured at the terminals of inductance $L_{p i j}$.

The current in both the two- and three-dimensional partial conductor crosssections is assumed to be uniform. 
An integral circuit equation solution can be set up for the solution of skin effect or current redistribution problem in the cross-section of two-dimensional conductors. The integral equation for the current density for each of a set of two-dimensional conductors is of the basic form

$\frac{J(\bar{r})}{\sigma}+\frac{j w \mu_{0}}{2 \pi} \int_{a} I_{n}\left|\bar{r}-\bar{r}^{\prime}\right| J\left(\overline{r^{\prime}}\right) d a^{\prime}=V(\bar{r})$

Where $\sigma$ is the conductivity and $J$ the current density in the conductor cross-section $a$. This can be transformed into an equivalent circuit in the form of Fig. 3 shown by discretization. The solution of the frequency-dependent inductance and resistance can be found from a set of equations that corresponds to the circuit shown or

$R I+s L_{p} I=V$

We can write equation (14) in state variable form in the time domain as

$$
\frac{d i}{d t}(t)=-L_{p}^{-1} R i(t)+L_{p}^{-1} V(t)
$$

An alternate solution method to the homogeneous equation can be stated as an eigenvalue problem,

$L_{p}^{-1} R I=\lambda I$

Where $\lambda$ is the eigenvalue.

A three-dimensional skin effect problem can be treated in a similar way by a three-dimensional integral equation. We call the equivalent circuits that result from the integral equation partial element equivalent circuits (PEEC). The solution in the time domain for the $L / R$ problems can be given in the form

$$
\left[\begin{array}{ll}
-A^{T} & R+L_{p} \frac{d}{d t} \\
0 & A
\end{array}\right]\left[\begin{array}{l}
V(t) \\
i(t)
\end{array}\right]=\left[\begin{array}{c}
0 \\
I s(t)
\end{array}\right]
$$

Where $I_{s}(t)$ are the forcing current sources and $A$ is the incidence matrix.

\section{ELECTRICAL MODELS AND ANALYSIS:}

Electrical circuit models are key to the analysis approach pursued here and several advantages result from this approach. The possible coupling among the sub-models is specified in terms of mutual capacitances and inductances.

The key transmission line model is shown in Fig. 4 as the voltage source model, which is based on the method of characteristic from the theory of partial differential equations. 


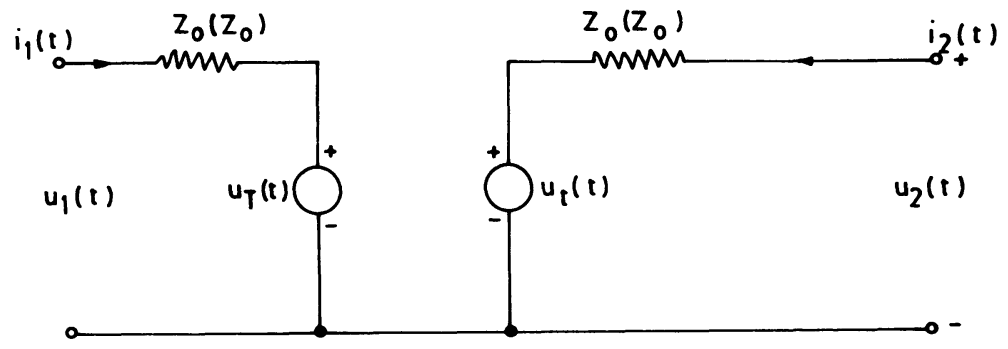

FIGURE 4 Voltage source model for transmission line

The reflected voltage in the Fig. 4 is

$U_{r}(t-\tau)=U_{2}(t)-Z_{0} i_{2}(t)$

while the transmitted voltage is

$U_{t}(t-\tau)=U_{1}(t)-Z_{0} i_{1}(t)$

Where $\tau$ is the delay and $Z_{0}$ the characteristic impedance

$Z_{0}=\sqrt{L / C}$

The lossless nature of this model restricts its applications and the inclusion of losses is also possible. For example, a resistance $R$ in series to the section of transmission line shown in Fig. 4 is accompanied by defining new impedances.

$Z_{0}^{+}=Z_{0}+R / 2$

and

$Z_{0}^{-}=Z_{0}-R / 2$

The new impedances in Fig. 4 are $Z_{0}^{+}$, as indicated and the voltage source equations, are

$U_{r}(t-\tau)=U_{2}(t)-Z_{0}^{-} i_{2}(t)$

$U_{t}(t-\tau)=U_{1}(t)-Z_{0}^{-} i_{2}(t)$

This model applies only to short resistance lines; extensions for long lines are also possible.

Another important transmission line problem that requires special attention is the propagation of signals on high performance on chip interconnections. As shown in Fig. 5, the silicon substrate represents a lossy ground return plane for the signals on the wire. It is known and can be shown also that for $\varepsilon_{o x}<\varepsilon_{s i}$ and $t_{o x}<t_{s i}$, the structure supports slow waves. 


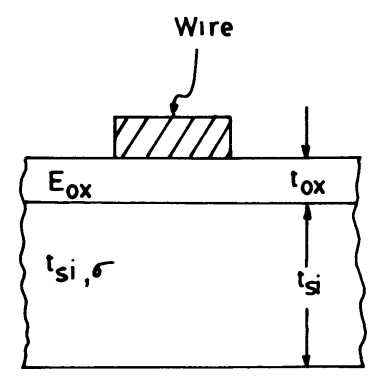

(a)

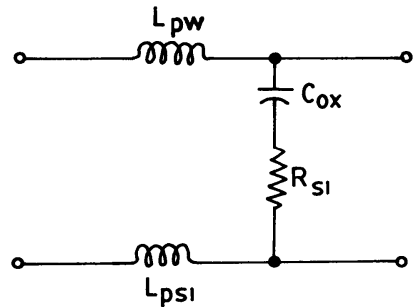

(b)

FIGURE 5 Wire on silicon and circuit model (a) Cross section of wire on $\mathrm{Si}$ and (b) Equivalent circuit model

A simple physical model for the phenomenon is given in Fig. 5 for a range of frequencies within the pulse spectrum: the capacitance of the equivalent transmission line is given in Fig. 5(b) by the oxide capacitances, which is large since $t_{o x}$ is small.

The incidence is large since the current penetration or skin effect in the silicon leads to a remote path, and a much larger delay $d=\sqrt{L C}$ results, which is very undesirable. Delays of $0.3 \mathrm{~ns} / \mathrm{cm}$ are not uncommon.

The PEECs are derived by summing all sources of electric field inside a conductor:

$E_{0}(\bar{\gamma}, t)=\frac{\bar{J}(\bar{\gamma}, t)}{\sigma}+\frac{\partial \bar{A}}{\partial t}(\bar{\gamma}, t)+\nabla V(\bar{\gamma}, t)$

Where $E_{0}$ represents an applied field. Both the vector and scalar potentials $A$ and $V$, respectively, are expressed in terms of integrals,

$$
\begin{aligned}
& \bar{A}(\bar{\gamma}, t)=\sum_{k=1}^{K} \frac{\mu}{4 \pi} \int_{V_{k}} \frac{1}{\left|\bar{\gamma}-\bar{\gamma}^{\prime}\right|} \bar{J}\left(\bar{\gamma}, t^{\prime}\right) d v^{\prime} \\
& V\left(\bar{\gamma}^{\prime}, t\right)=\sum_{k=1}^{K} \frac{1}{4 \pi \varepsilon} \int_{V_{k}} \frac{1}{\left|\bar{\gamma}-\bar{\gamma}^{\prime}\right|} q\left(\bar{\gamma}, t^{\prime}\right) d v^{\prime}
\end{aligned}
$$

Where $k$ conductors are involved in the system.

Partial capacitances are required to represent surfaces that become nonequipotentials under time domains or high frequency excitation. Fig. 6 shows a cell configuration. The surface cells are capacitance cells, which are electrically disconnected by an infinitesimal gap. Thus, the partial capacitance between them is finite and it can be easily computed. 


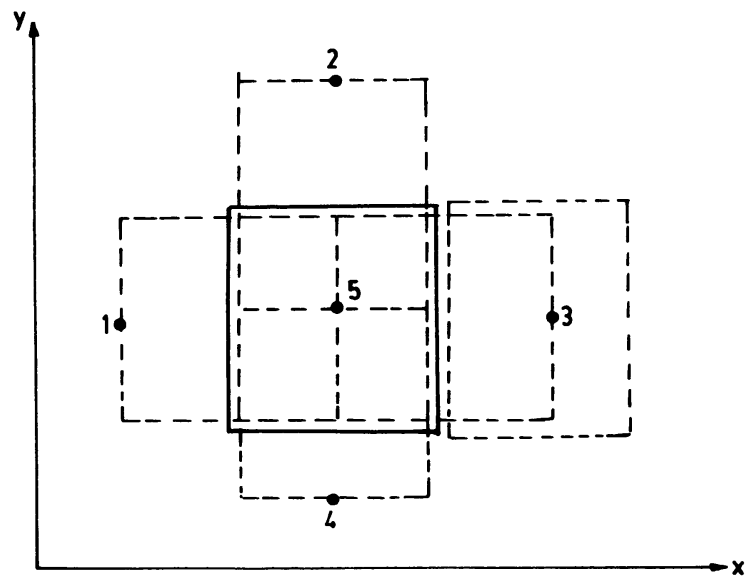

FIGURE 6 Cell structure for PEEC

\section{STUDY OF FINITE ELEMENT APPROACH TO DEVICE MODELLING-A NOVEL APPROACH}

The Mesh

The use of triangular elements for discretization of the simulation domain has the following drawbacks:

a) A general mapping from a triangular parent mesh to a complex geometry can be found, but local refinement of a grid is rather difficult.

b) Loosening of the grid spacing is almost impossible.

\section{DISCRETIZATION ERROR FOR TRIANGULAR MESH-}

This is given by $(1 / \sin a)$ where $a$ is the smallest angle of the triangle. Therefore, we should try to avoid obtuse triangles as far as possible.

A5-node element-[Fig. 7]

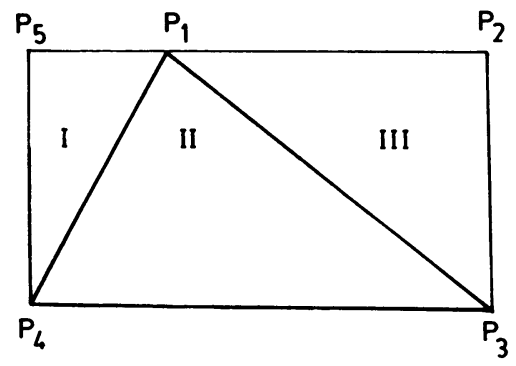

FIGURE 7 A $S$-node element 
As long as the aspect ratio of the rectangular structure is between 0.5 and 2 , no obtuse triangle is formed.

\section{INITIAL GRID-}

It is established using information on the device geometry and the doping profile. Boundary zones and interior regions are each marked with different attributes.

\section{PRE-INITIAL GRID-}

It consists of the absolutely essential elements, which contain nodes on every boundary and in every interior region.

\section{THE SHAPE FUNCTIONS-}

A central task in the numerical solution of semiconductor equations is the preservation of current continuity parallel to element edges and the preconditioning of the system in a way that the exponential variation of carrier densities will be represented exactly by the discrete system.

a) Rectangle-

Making the following assumptions-

(i) Exponential variations of carrier densities.

(ii) No recombination.

We can write the continuity equation as

$$
\text { divergence }(J)=0
$$

where $J=\exp (\psi) \operatorname{grad}(\xi)$

$\psi=$ a known potential fucntion

$\xi=\exp \left(-\phi_{n}\right)$

Substitute (25) in (24)

$$
\begin{aligned}
0 & =\operatorname{Div}(J)=\operatorname{Div}(\exp (\psi) \operatorname{grad}(\xi)) \\
& =\operatorname{grad}(\exp (\psi) \operatorname{grad}(\xi)+\exp (\psi) \operatorname{div}(\operatorname{grad}(\xi))) \\
& =\exp (\psi)(\operatorname{grad}(\psi) \operatorname{grad}(\xi)+\operatorname{div}(\operatorname{grad}(\xi)))
\end{aligned}
$$

$[$ Since $\nabla \cdot(f \bar{G})=\nabla f \cdot \vec{G}+f \nabla \cdot \vec{G}]$

or

$$
\frac{\partial \psi}{\partial x} \frac{\partial \xi}{\partial x}+\frac{\partial^{2} \xi}{\partial x^{2}}=-\frac{\partial \psi}{\partial y} \frac{\partial \xi}{\partial y}-\frac{\partial^{2} \xi}{\partial y^{2}}
$$


It has been possible to find an analytical solution under the additional assumption of a constant field, i.e., linearity of $\psi$ in $x$ and $y$. Although this is not totally consistent with bilinear shape functions for $\psi$, the resulting solution is still worth close consideration.

By separation of variables

$\xi=E(x) H(y)$

This leads to two independent ordinary differential equations of second order

$E^{\prime \prime}+\frac{\partial \psi}{\partial x} E^{\prime}-\lambda E=0$

$H^{\prime \prime}+\frac{\partial \psi}{\partial y} H^{\prime}+\lambda H=0$

With the solution for $\lambda=0$

$E=C_{1}+C_{2} \exp \left(\frac{-\partial \psi}{\partial x} x\right)$

$H=K_{1}+K_{2} \exp \left(\frac{-\partial \psi}{\partial y} y\right)$

Calculating the product and using the boundary conditions to evaluate constants

$$
\begin{aligned}
\xi(x, y)= & \xi_{1} \alpha_{1}(x, y)+\xi_{2} \alpha_{2}(x, y)+\xi_{3} \alpha_{3}(x, y) \\
& +\xi_{4} \alpha_{4}(x, y)
\end{aligned}
$$

The $\xi_{1}, \xi_{2}, \xi_{3}, \xi_{4}$ are the values of the functions at the 4 nodes and the $\alpha_{i}$ are the basic functions

$$
\begin{aligned}
& \alpha_{1}(x, y)=\left[1-\phi_{1}(x, y)\right]\left[1-\phi_{2}(x, y)\right] \\
& \alpha_{2}(x, y)=\phi_{1}(x, y)\left[1-\phi_{2}(x, y)\right] \\
& \alpha_{3}(x, y)=\phi_{1}(x, y) \phi_{2}(x, y) \\
& \alpha_{4}(x, y)=\left[1-\phi_{1}(x, y)\right] \phi_{2}(x, y) \\
& \text { for } \exp \left(-\phi_{n}\right) \\
& \beta_{1}(x, y)=\left[1-\sigma_{1}(x, y)\right]\left[1-\sigma_{2}(x, y)\right] \\
& \beta_{2}(x, y)=\sigma_{1}(x, y)\left[1-\sigma_{2}(x, y)\right] \\
& \beta_{3}(x, y)=\sigma_{1}(x, y) \sigma_{2}(x, y) \\
& \beta_{4}(x, y)=\left[1-\sigma_{1}(x, y)\right] \sigma_{2}(x, y)
\end{aligned}
$$


For $\exp \left(\phi_{p}\right)$ where

$$
\begin{aligned}
& \phi_{1}(x, y)=f\left(x-\frac{\partial \psi}{\partial x}\right) \\
& \phi_{2}(x, y)=f\left(y-\frac{\partial \psi}{\partial y}\right) \\
& \sigma_{1}(x, y)=f\left(x, \frac{\partial \psi}{\partial x}\right) \\
& \sigma_{2}(x, y)=f\left(y, \frac{\partial \psi}{\partial y}\right)
\end{aligned}
$$

With $f(x, a)=(\exp (a x)-1)[\exp (a)-1]$

With the appropriate scaling factors, this derivation holds valid for any rectangle. This implies that physically plausible shape functions can only be derived from a solution of the Dirichlet problem. Formulation of shape functions in closed form were only possible if a constant field was assumed in each triangle.

With these basic functions, one can derive expressions in the unit square for electron concentration-

$n(x, y)=n_{1} B_{1}+n_{2} B_{2}+n_{3} B_{3}+n_{4} B_{4}$

and for the electron current density

$$
\begin{array}{r}
J_{n x}(x, y)=\left(1-\sigma_{2}\right)\left[B\left(\frac{\partial \psi}{\partial x}\right) n_{2}-B\left(\frac{-\partial \psi}{\partial x}\right) n_{1}\right]+\sigma_{2}\left[B\left(\frac{\partial \psi}{\partial x}\right) n_{3}\right. \\
\left.-B\left(\frac{-\partial \psi}{\partial x}\right) n_{4}\right] \\
J_{n y}(x, y)=\left(1-\sigma_{1}\right)\left[B\left(\frac{\partial \psi}{\partial y}\right) n_{4}-B\left(\frac{-\partial \psi}{\partial y}\right) n_{1}\right]+\sigma_{2}\left[B\left(\frac{\partial \psi}{\partial y}\right) n_{3}\right. \\
\left.-B\left(\frac{-\partial \psi}{\partial y}\right) n_{1}\right]
\end{array}
$$

and similarly for the hole concentration

$P(x, y)=P_{1} a_{1}+P_{2} a_{2}+P_{3} a_{3}+P_{4} a_{4}$ 
and for the hole current density

$$
\begin{aligned}
J_{p x}(x, y)= & \left(1-\phi_{2}\right)\left[B\left(\frac{\partial \psi}{\partial x}\right) P_{2}-B\left(\frac{-\partial \psi}{\partial x}\right) P_{1}\right] \\
& +\phi_{2}\left[B\left(\frac{\partial \psi}{\partial x}\right) P_{3}-B\left(\frac{-\partial \psi}{\partial x}\right) P_{4}\right] \\
J_{p y}(x, y)= & \left(1-\phi_{1}\right)\left[B\left(\frac{\partial \psi}{\partial y}\right) P_{4}-B\left(\frac{-\partial \psi}{\partial y}\right) P_{1}\right]+\phi_{1}\left[B\left(\frac{\partial \psi}{\partial Y}\right) P_{3}\right. \\
& \left.-B\left(\frac{-\partial \psi}{\partial y}\right) P_{2}\right]
\end{aligned}
$$

Where $B(a)$ is the Bernoulli function.

$B(a)=a /(\exp (a)-1)$

Case (i)-For a high electric field the current generates into a pure field current

$J_{n x}=\frac{\partial \psi}{\partial x}\left(n_{1}(1-y)+n_{4} y\right)$

Case (ii)-For no field, the current only consists of diffusion current

$J_{n y}=\left(1-\sigma_{2}\right)\left(n_{2}-n_{1}\right)+\sigma_{2}\left(n_{3}-n_{1}\right)$

for $\partial \psi / \partial x \gg 0$ and $\partial \psi / \partial y=0$

b) Transition Band

This macroelement is partitioned into 3 elements in each of these triangles; we take the current density to be constant. Thus, the component of the current density parallel to the edge that connects vertices $i$ and $j$ becomes

$J_{i j}=\left[n_{i} B\left(\psi_{i}-\psi_{j}\right)-n_{j} B\left(\psi_{j}-\psi_{i}\right)\right] l_{i j}$

where $l_{i j}$ is the length of that edge

Because of non-linearity of the Bernoulli function, the current expressions are no longer additive.

$J_{i j}=J_{i k}+J_{k j}$

Since the main point of interest is continuity of the current component parallel to the edges, one has to give preference to the components in the $x$ and $y$ directions. This is obviously trivial. Difficulty arises in non-rectangular triangles. Although any arbitrary triangle can be linearly transformed into a rectangular triangle, the 
current would no longer transform correctly. There exists a number of possibilities to calculate the $y$ component. The most promising is the super-position of $J_{14}$ and $J_{13}$.

\section{FINITE ELEMENT SEMICONDUCTOR DEVICE SIMULATIONS-}

The FEDSS program simulates semiconductor process in two dimensions. The process steps to be modelled include ion implantation, oxidation/drive-in, chemical predeposition through the surface, and oxide depositions.

(a) Ion implantation-This is a method of introducing impurities into semiconductor materials. Ion implantation steps in FEDSS may be specified in two ways-

(i) To specify the implant energy in $\mathrm{KeV}$ and the dose in ions per square cm.

(ii) To specify the distribution parameter and dose.

Implants can be windowed to place them in a region defined by a mask. The effects of a mask and the mask edge are taken into account. Either vertical mask edges or arbitrary edges can be specified. Where required, exponential tails are added to the ion-implanted distributions. If multiple layers are present, the stopping power of all materials is assumed to be the same.

(b) Drive-in-Given an initial concentration distribution, the diffusion equation is given by Fick's second law

$$
\operatorname{div}(D \operatorname{grad} C)=\frac{\partial C}{\partial t}
$$

is simulated for a given number of minutes at a given temperature. The diffusivity $D$ can be concentration dependent if the concentration is high.

The specific form of the diffusion equation solved by FEDSS is

$$
\operatorname{div}(D \text { eff } \operatorname{grad} C)=\frac{\partial C}{\partial t}
$$

Equation (31) can be used to derive diffusion flux for donor

$$
J=-D \operatorname{grad} N_{D}+\mu E N_{D}
$$

Using the Einstein equation, we can write

$$
J=-D \operatorname{grad} N_{D}-D N_{D} \operatorname{grad}\left(l_{n}\left(n / n_{i}\right)\right)
$$

Since $\left(n / n_{i}\right)$ is a function of $N_{D}$, we can apply a chain rule.

$J=-D \operatorname{grad} N_{D} \cdot-\left(D N_{D}\right) /\left(n / n_{i}\right)\left(\partial\left(n / n_{i}\right) / \partial N_{D}\right)$ grade $N_{D}$

Setting $\left.D_{\text {eff }}=D\left(1+N_{D}\right) /\left(n / n_{i}\right)\right)\left(\partial\left(n / n_{i}\right) / \partial N_{D}\right)$ 
Assuming total flux for As is $C_{T}$, including the effect of $E$ on the charged vacancy:

$-J_{C T}=D_{\text {eff }} \partial C_{T} / \partial x$

From calculus, the L.H.S. of equation (30)

$I(C)=\int_{A}(D / 2) \operatorname{grad}\left(C^{2}\right)-C(\partial C / \partial t) d A$

while R.H.S. is

$\partial C / \partial t=\left(C(t)-C(t-\Delta t) / \Delta t=\left(C_{i}-C_{i}-1\right) / \Delta t\right.$

Now we can write

$(A)\left(C_{i}\right)=(B)\left(C_{i-1}-C_{i}\right) / \Delta t$

(c) Oxidation-Simulation of the growth of “Bird's Beak" region is easier. The parabolic rate constant $B$ is given by

$B=\left(2 D_{\text {eff }} C^{*}\right) / N_{i}$

The coefficient $(B / A)=(k h) /(k+h) X\left(C^{*} / N_{i}\right)$. If $h$ is very high, then $D_{\text {cff }}$ through $\mathrm{SiO}_{2}$ is given by $k=\left(2 D_{\text {eff }} / A\right)$

Where $D_{\text {eff }}=\left(B N_{1}\right) /\left(2 C^{*}\right)$

During oxidation, in addition to impurities diffusing, $\mathrm{O}_{2}$ is diffusing through $\mathrm{SiO}_{2}$ with an effective diffusivity $D_{\text {eff }}$. The amount of oxygen in the silicon is accumulated and when a sufficient quantity has been collected, $\mathrm{S}_{\mathrm{i}} \mathrm{O}_{2}$ molecules are formed.

(d) Low-Temperature Oxide Deposition-Here impurities are introduced after an oxidation step and the redistribution in subsequent temperature cycles is to be studied. In this step, doped or undoped oxide is deposited on the silicon surface on the top of any existing oxide. The amount of oxide deposited is the interior of the rectangle whose vertices are specified. If the deposited oxide is to be doped, then the impurity type and concentration is specified.

(e) Chemical predeposition through the surface:-The deposition step is similar to the drive-in step, except that certain nodes, specified by the user, have their concentration fixed during this step. The predeposition step models the introduction of an impurity element from a constant source at the Si-surface. 


\section{PRESENTED METHOD FOR VLSI INTERCONNECTIONS MODELLING:}

\section{CONCEPT OF SPIDERS}

The assembling elements used are referred to as "Spider Cells". These are sets of edges that are incident in a given mode. The size of spider cells, as well as their shape, can be selected arbitrarily depending on the accuracy desired.

\section{CAPACITANCE CO-EFFICIENTS OF THREE-DIMENSIONAL MULTI-CONDUCTOR SYSTEMS}

The integral equation relating the electrostatic potential $V(p)$ to the charge density $\sigma(q)$ in a chargeless three dimensional dielectric medium may be written as

$V(p)=\int_{\text {all charge }} G(p, q) \sigma(q) d q$

where $G(p, q)=$ Green's function = physically the potential at a point in space $p(x, y, z)$ due to a unit point charge at another point $q\left(x^{\prime}, y^{\prime}, z^{\prime}\right)$.

To compute the parasitic capacitances, we have to calculate the relations between the charge $Q_{i}$ on each conductor and its potential $V_{i}$. Therefore, we have to invert equation (40).

\section{DISCRETIZATION OF CHARGE DISTRIBUTION}

Discretization is done to reduce the Green's integral equation to a discrete problem.

To achieve high efficiency, a finite element scheme is proposed in which charge is concentrated along edges that form a cage enclosing the conductor, as shown in the Fig. 8. The advantage is that although the dimensionality of the charge is reduced from two to one, still the results are as accurate as with a surface distribution.

As shown in Fig. 9, there is a spider function associated with each node. This is concentrated on the edges of the spider, and is linear. It has a normalized value at the node representing total charge 1 and is 0 at adjacent nodes. The charge distribution on the conductor $j$ will then be described by a linear combination of $N_{j}$ spider functions with appropriate weights. The result is a piece-wise linear charge distribution function concentrated along the network of edges.
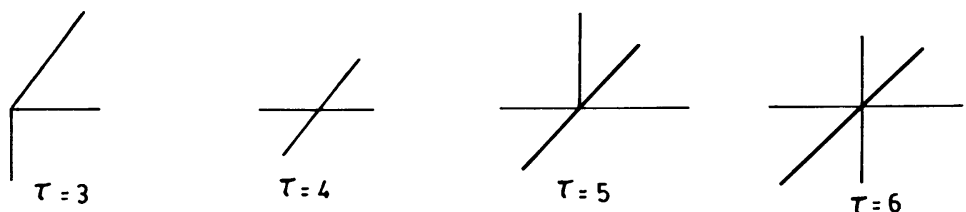

FIGURE 8 Number of edges for different spiders for discretization 


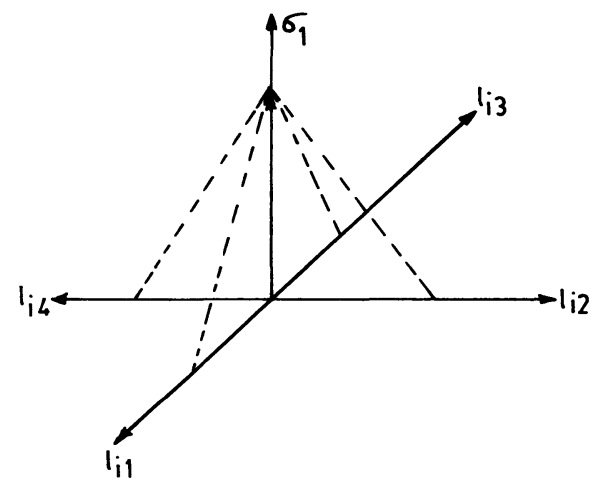

FIGURE 9 A spider shape function $(T=4)$

The shape function associated to the $i$ th spider is given in the slope intercept form by

$f_{i}=\sum_{k=1}^{\tau} f_{i k}=\sum_{k=1}^{\tau} A_{i k}\left(l_{i k}-B_{i k}\right)$

where $\tau=3,4,5$ or $6 ; i=1,2, \ldots, N_{j}$ and $0 \leq l_{i k} \leq B_{i k}$ where $l_{i k}$ is the running coordinate along the spiders $k$ th edge

$B_{i k}=$ intercept point

$A_{i k}=$ slope

$\tau=$ number of legs of a spider

$=$ also the value of $\tau$ represents the spider shape.

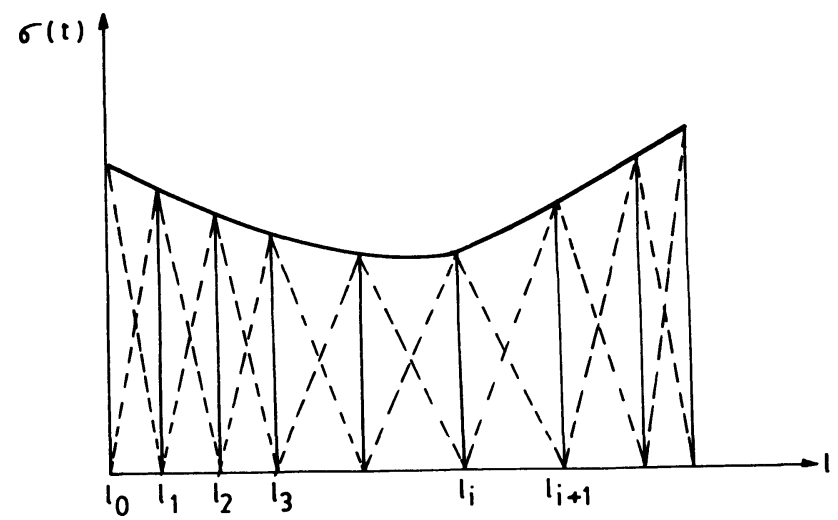

FIGURE 10 The piecewise linear charge density on each edge on both top and bottom of a single contour 
$A_{i k}$ and $B_{i k}$ can be determined by boundary conditions of the spider's cells and the normalization condition. The boundary conditions are for $l_{i k}=0$ all $1 \leq k \leq \tau$

$f_{i}=f_{i 0}$

for $l_{i k}=B_{i k}$ and all $1 \leq k \leq \tau$

$f_{i}=0$

The normalization condition is

$\sum_{k=1}^{\tau} \int f_{i} d l_{i k}=1$

using equations (41), (42), (43) and (44)

$A_{i k}=-2 /\left(B_{i k}\left(\sum_{j=1}^{\tau} B_{i j}\right)\right)$

As a consequence, the charge distribution $\sigma_{j(q)}$ over the edges can be written as

$\sigma_{j(q)}=\sum_{i=1}^{N_{j}} \alpha_{j i} f_{j i}(q)$

where $f_{j i}(q)$ is the $i$ th of $N_{j}$ spider's shape functions in the $j$ th conductor and $\alpha_{i j}$ are the unknown coefficients to be determined.

\section{GREEN'S FUNCTION}

The three-dimensional Green's function for the Laplacian operator in a homogeneous dielectric is

$G(p, q)=1 /(4 \pi \varepsilon(-p-q))$

where $G(p, q)$ represents the electrostatic potential at a point $p$ due to a unit point charge at point $q$.

As a special case, when both the potential node and charge node are in the $\mathrm{SiO}_{2}$ layer or in the protective coating above the conductor with the same 
permittivity as oxide, the Green's function can be written as

$$
\begin{aligned}
& G(p, q)=\frac{1}{4 \pi \varepsilon_{1}}\left\{\begin{array}{l}
\frac{1}{\sqrt{z_{1}^{2}+\rho^{2}}} \\
-\frac{1}{\sqrt{\left(2 z^{\prime}+z_{1}\right)^{2}+\rho^{2}}}
\end{array} \quad+\sum_{n=0}^{\infty}(-1)^{n} k^{n+1}\right. \\
& \times\left[\frac{1}{\sqrt{\left[2(n+1) d-\left(2 z^{\prime}+z_{1}\right)\right]^{2}+\rho^{2}}}-\frac{1}{\sqrt{\left.\left[2(n+1) d+z_{1}\right)\right]^{2}+\rho^{2}}}\right. \\
& +\frac{1}{\sqrt{\left[\left(2(n+1) d+2 z^{\prime}+z_{1}\right]^{2}+\rho^{2}\right.}}-\frac{1}{\sqrt{\left[\left(2(n+1) d+z_{1}\right]^{2}+\rho^{2}\right.}}
\end{aligned}
$$

where $\varepsilon_{1}$ denotes the dielectric constant of $\mathrm{SiO}_{2} . d$ denotes the thickness of $\mathrm{SiO}_{2}$ and $Z_{1}=Z-Z^{\prime}$

$$
\begin{aligned}
& \rho=\left(\left(x-x^{\prime}\right)^{2}+\left(y-y^{\prime}\right)^{2}\right)^{1 / 2} \\
& k=\left(\varepsilon_{1}-\varepsilon_{2}\right) /\left(\varepsilon_{1}+\varepsilon_{2}\right)
\end{aligned}
$$

\section{SOLUTION OF INTEGRAL EQUATION}

For an $M$-conductor system, we can write

$\phi_{j}(p)=\sum_{i=1}^{M} \int_{S_{i}} G(p, q) \sigma_{i}(q) d S_{i}(q) \quad j=1,2, \ldots, M$

where $\sigma_{i}(q)$ denotes the charge density on the edges of the $i$ th conductor surface $S_{i}$. Also, the potential $\phi_{w}(p)$ at the $w$ th node is determined as

$\phi_{w}(p)=\sum_{j=1}^{M} \sum_{i=1}^{M} \alpha_{j i} F_{j i}(p) \quad w=1,2, \ldots, L$

where $F_{j i}(p)=\int G(p, q) f_{j i}(q) d l_{j i}(q)$

jith spider 
and $L$ is the total number of spider cells in the system.

$L=\sum_{j=1}^{M} N_{j}$

In the matrix notation, equation (47) written as

$F A=J \phi$

where $A^{T}=\left(\alpha_{11}, \ldots, \alpha_{1 N}, \alpha_{21}, \ldots, \alpha_{2 N_{2}}\right)$

$\left.\alpha_{M_{1}}, \ldots, \alpha_{M N_{m}}\right)$

It denotes the set of co-efficients to be determined. $F$ is the $(L \times L)$ matrix of the single integrals. $\phi^{T}=\left(\phi_{1}, \phi_{2}, \ldots \phi_{M}\right)$ is the set of the $M$ distinct potentials of the $M$ conductors. $J=(L \times M)$ incidence matrix of modes and conductors in which $J_{j i}=1$ if conductor $i$ contains node $j$.

\section{CAPACITANCE MATRIX}

The capacitance matrix $C$ is defined as $Q=C \phi$, where $Q$ is the vector of total charges on the conductors. As has been written above

$Q=J^{T} A$

Also, since $F A=J \Phi$

Therefore, $A=F^{-1} J \Phi$

Therefore, we can write

$Q=J^{T} F^{-1} J \Phi=C \Phi$

From the above equation, we can write

$C=J^{T} F^{-1} J$

The above equation can be solved for capacitance matrix by any standard technique.

\section{NUMERICAL CONSIDERATIONS}

The method for calculation of $F$ matrix is given below. If $j \neq i$, the integrals for the relative nodes $p$ and $q$ is $F(p)=\sum_{k=1}^{\tau} I_{k}$ where $I_{k}=\int_{l_{k}=0}^{\beta_{k_{G}(p, q) f_{k}} d I_{k} \text {. }}$ Evaluation of integrations $I_{k}$ can be narrowed down to evaluation of two functions. 
They are

$$
\begin{aligned}
& n_{k}=\xi A_{k}\left\{\sqrt{d_{k}^{2}+\rho_{k}^{2}}-\sqrt{R_{k}^{2}+\rho_{k}^{2}}-\left|d_{k}\right|\right\} l_{n} \\
& \frac{\left|d_{k}\right|+\sqrt{d_{k}^{2}+\rho_{k}^{2}}}{\left|R_{k}\right|+\sqrt{R_{k}^{2}+\rho_{k}^{2}}} \\
& \gamma_{k}=\xi A_{k}\left\{\sqrt{U_{k}^{2}+\xi_{k}^{2}}-\sqrt{U_{k^{2}}+\xi_{K^{2}}}\right\}-U_{k}+\xi_{n} \\
& \frac{U_{k}+\sqrt{U_{k^{2}}+\xi_{k^{2}}}}{v_{k}+\sqrt{v_{k}^{2}+\xi_{k^{2}}}}
\end{aligned}
$$

where with reference to Fig. 11.

$\xi=\left\{\begin{array}{l}\frac{1}{4 \pi \varepsilon_{1}} \text { for the first two terms for } G(p, q) \text { in equation }(46) \\ \frac{(-1)^{n} k^{(n+1)}}{4 \pi \varepsilon_{1}} \text { for the series terms for the expression of } G(p, q) \text { in equation }(46)\end{array}\right.$

$d_{k}=\beta_{k}+R_{k}$

$R_{k}=\left\{\begin{array}{l}x(q)-x(p) \text { if integral is in } x \text { direction } \\ y(q)-y(p) \text { if integral is in } y \text { direction } \\ z(q)-z(p) \text { if integral is in } z \text { direction }\end{array}\right.$

$U_{k}=\beta_{k}^{z}+V_{k}$

$V_{k}=z(q)+z(p)$ and

$\xi k^{2}=(x(q)-x(p))^{2}+(y(q)-y(p))^{2}$

Where $(x(q), y(q), z(q))$ and $(x(p), y(p), z(p))$ denote the co-ordinates of point $q$ and $p$, respectively. $\beta_{k}^{z}$ denotes the intercept in the $z$-direction.

If the integral is in the $x$-direction, as shown in Fig. 12:

$\rho_{k}^{2}=\Delta y^{2}+(z(p)-z(q))^{2}$

for the first term of $G(p, q)$ in equation (46). For the second term in equation (46),

$\rho_{k}^{2}=\Delta y^{2}+(z(p)+z(q))^{2}$ 


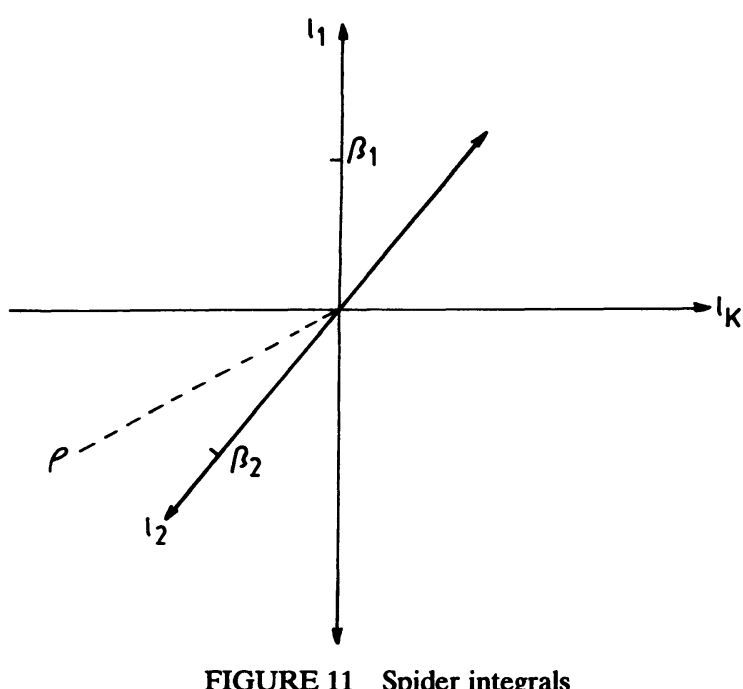

FIGURE 11 Spider integrals

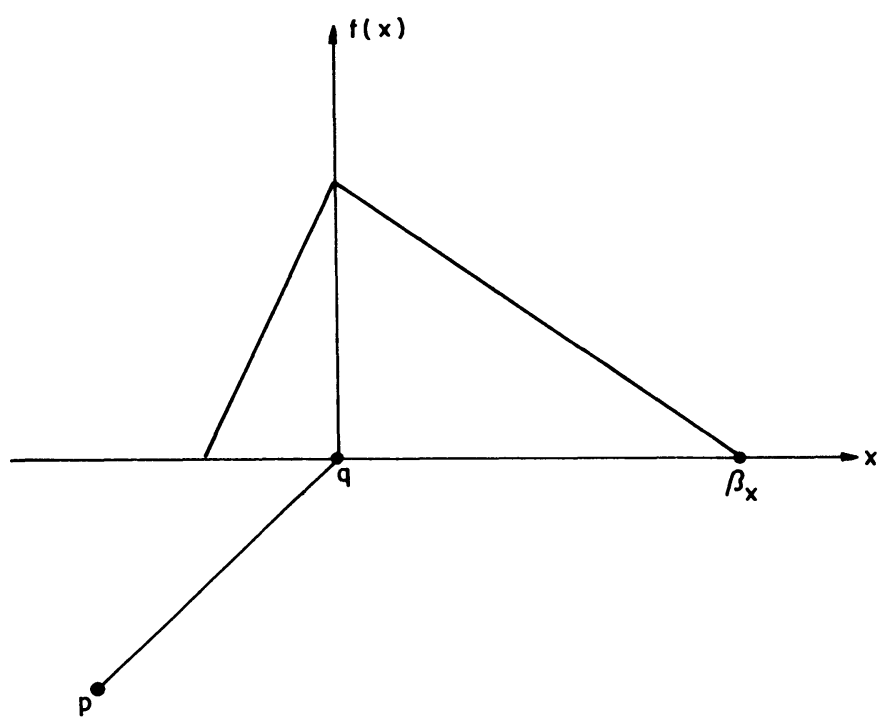

FIGURE 12 Integral in the $x$-direction

For the third term in equation (46)

$\rho_{k}^{2}=\Delta y^{2}+(2(n+1) d+z(p)-z(q))^{2}$

for the fourth term in equation (46)

$\rho_{k}^{2}=\Delta y^{2}+(2(n+1) d+z(p)-z(q))^{2}$ 
For the fifth term in equation (46)

$\rho_{k}^{2}=\Delta y^{2}+(2(n+1) d+z(p)+z(q))^{2}$

for the sixth term in equation (46)

$\rho_{k}^{2}=\Delta y^{2}+(2(n+1) d-z(p)+z(q))^{2}$

Where $\Delta y=y(p)-y(q)$.

Similar denotations of $\rho_{k}^{2}$ can be drawn in other directions. Both $n_{k}$ and $\gamma_{k}$ can be solved by subroutine.

\section{RESULTS AND CONCLUSIONS:}

A program called SPIDER was developed with the algorithm given in Fig. 13. The dimensions chosen are $1=10 \mu \mathrm{m}, w=5 \mu \mathrm{m}, t=1 \mu \mathrm{m}, h=2 \mu \mathrm{m}$ and spacing $s=5 \mu \mathrm{m}, \varepsilon=3.9 \times 8.854 \mathrm{E}-10 \mathrm{~F} / \mathrm{cm}$ (for dielectric)

$\varepsilon_{0}=8.854 \mathrm{E}-10 \mathrm{~F} / \mathrm{cm}$

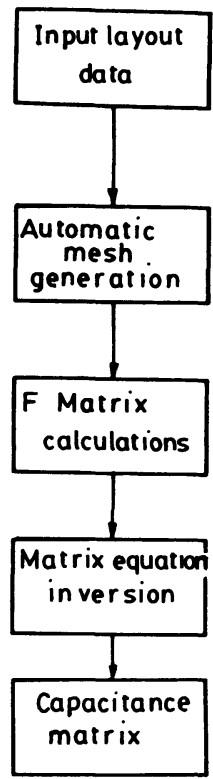

FIGURE 13 Spider flowchart 
The program calculates the capacitances $C_{11}$ and $C_{22}$. The computational complexity is essentially determined by:

1. The number of spider cells to be determined, which is equal to the number of nodes in the mesh.

2. This number also determines the number of Green's function calculations.

3. The complexity of Green's function is itself the complexity of method.

4. The present method achieves its efficiency in the drastic reduction of CPUtime for evaluation of Green's function.

5. Also by increasing the number of terms in the series representing $G(p, q)$, we can reduce the error involved in the calculation of capacitance.

This method is both less complex and more general, without unduly increasing the storage requirement for the coefficient matrix. A mathematical difficulty with our method is that the elastance matrix obtained is not exactly symmetrical. A doubling of the dimension of the integral is avoided, at the cost of a symmetry. When the grid is sufficiently small, the errors are also small.

\section{REFERENCES}

1. B. H. McDonald, M. Friedman, and A. Wexler, "Variational solution of Integral Equations," IEEE Trans. Microwave Theory Tech., MTT-22, 237-248 (1974).

2. Z. Q. Ning, P. Dewilde, and F. L. Neerhoff, "Capacitance coefficients for VLSI multi-level metallization lines," IEEE Trans Electron Devices, Vol. ED-34, pp. 644-649, Mar. 1987.

3. C. D. Tayler, G. M. Eikhouri, and T. E. Wade, "On the parasitic capacitances of multilevel parallel metallization lines," IEEE Trans. Electron Devices, Vol. ED-32, Nov. 1985.

4. W. H. Dierking and J. D. Bastian, "VLSI parasitic capacitance determination by flux tubes," IEEE Circuits Syst. Mag., pp. 11-18 Mar. 1982.

5. Albert E. Ruehli, "Survey of computer Aided Electrical Analysis of Integrated Circuit Interconnections," IBM J. of Research and development, Vol. 23, Number 6, Nov., 1979, pp. 626-639.

6. Zhen-Qiu Ning and Patrick M. Dewilde, "Spider: Capacitance Modelling for VLSI Interconnections,” IEEE Trans. on CAD, Vol. 7, No. 12, Dec. 1988, pp. 1221-1228.

7. Roberto Guerrieri and Marsimo Rudan, "Optimization of the Finite-Element Solution of the Semiconductor Device Poirson Equation," IEEE Trans. on Electron Devices, Vol. ED-30, No. 9, Sept., 1983, pp. 1097-1102.

8. Kevyna Salsburg and Howard H. Hausen, "FEDSS-Finite Element Diffusion-Simulation Systems," IEEE Trans. on Electron Devices, Vol. ED-30, No. 9., Sept., 1983, pp. 1004-1010.

9. R. C. Frye, "Analysis of the Trade-offs Between Conventional and super conducting Inter connections” IEEE Circuits and Devices Magazine, May, 1989, pp. 27-32.

10. A. H. Zemanian, Fellow, IEEE, "A Finite-Difference Procedure for the exterior Problem Inherent in capacitance computations for VLSI Interconnections," IEEE. Trans. on Electron Devices, Vol. 35, No. 7, July, 1988, pp. 985-992. 

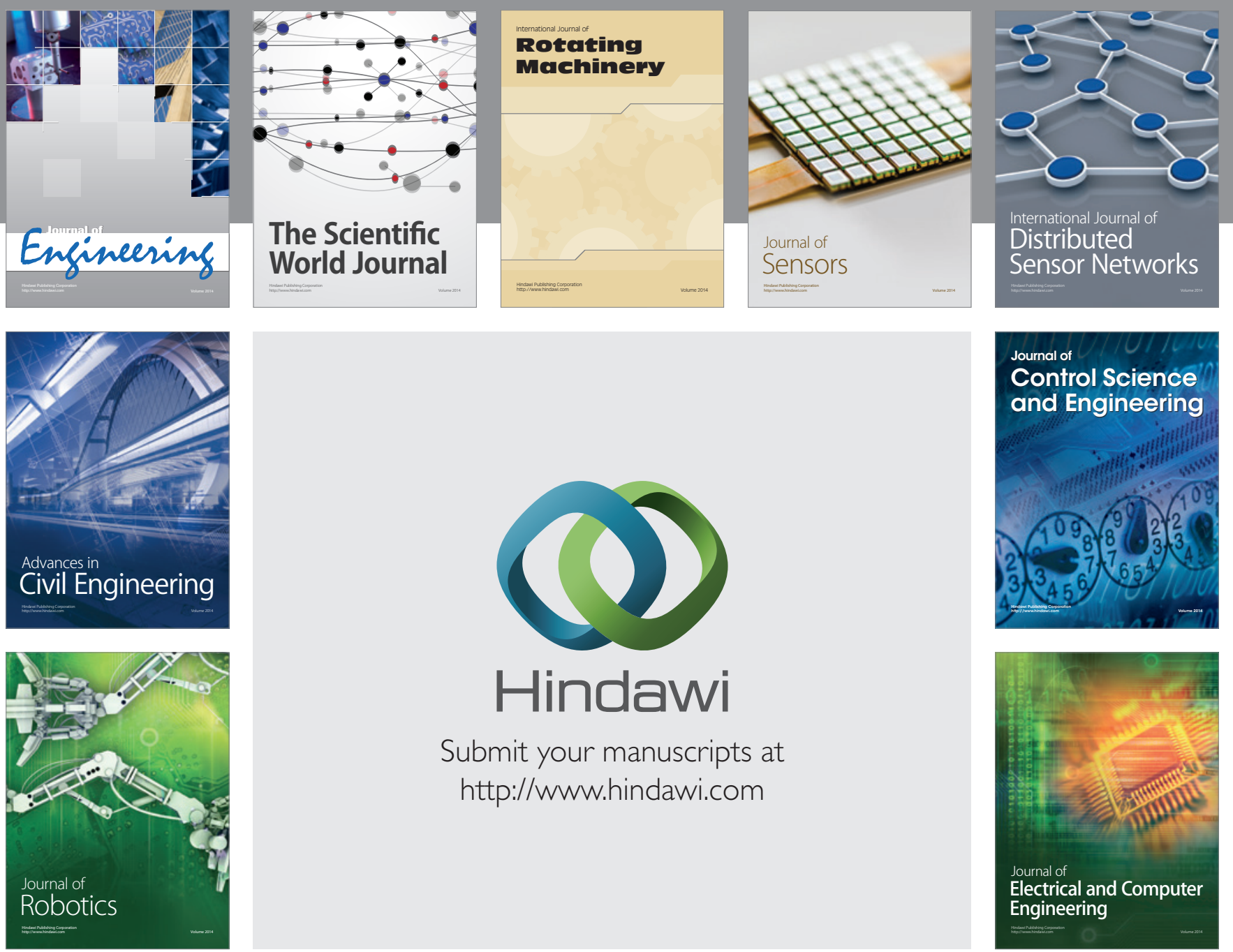

Submit your manuscripts at

http://www.hindawi.com
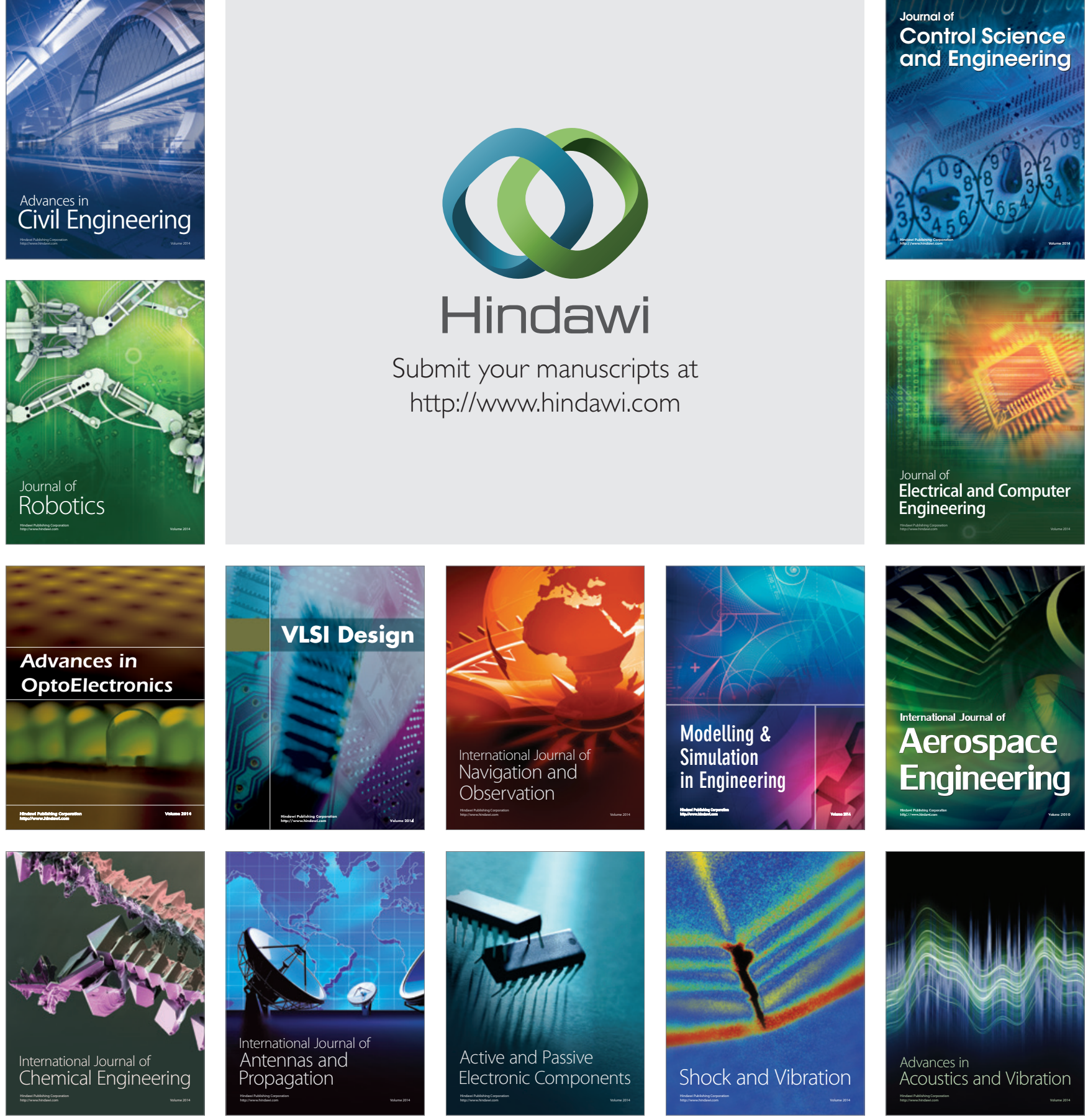